\title{
On the analysis of the contact angle for impacting droplets using a polynomial fitting approach
}

\author{
Miguel A. Quetzeri-Santiago ${ }^{1,2}$ · J. Rafael Castrejón-Pita ${ }^{2}$ Alfonso A. Castrejón-Pita ${ }^{1}{ }^{\circledR}$
}

Received: 13 February 2020 / Revised: 27 April 2020 / Accepted: 3 May 2020 / Published online: 2 June 2020

(c) The Author(s) 2020

\begin{abstract}
Practical considerations on the measurement of the dynamic contact angle and the spreading diameter of impacting droplets are discussed in this paper. The contact angle of a liquid is commonly obtained either by a polynomial or a linear fitting to the droplet profile around the triple-phase point. Previous works have focused on quasi-static or sessile droplets, or in cases where inertia does not play a major role on the contact angle dynamics. Here, we study the effect of droplet shape, the order of the fitting polynomial and the fitting domain, on the measurement of the contact angle on various stages following droplet impact where the contact line is moving. Our results, presented in terms of the optical resolution and the droplet size, show that a quadratic fitting provides the most consistent results for a range of various droplet shapes. As expected, our results show that contact angle values are less sensitive to the fitting conditions for the cases where the droplet can be approximated to a spherical cap. Our experimental conditions include impact events with liquid droplets of different sizes and viscosities on various substrates. In addition, validating past works, our results show that the maximum spreading diameter can be parameterised by the Weber number and the rapidly advancing contact angle.
\end{abstract}

\section{Graphic abstract}

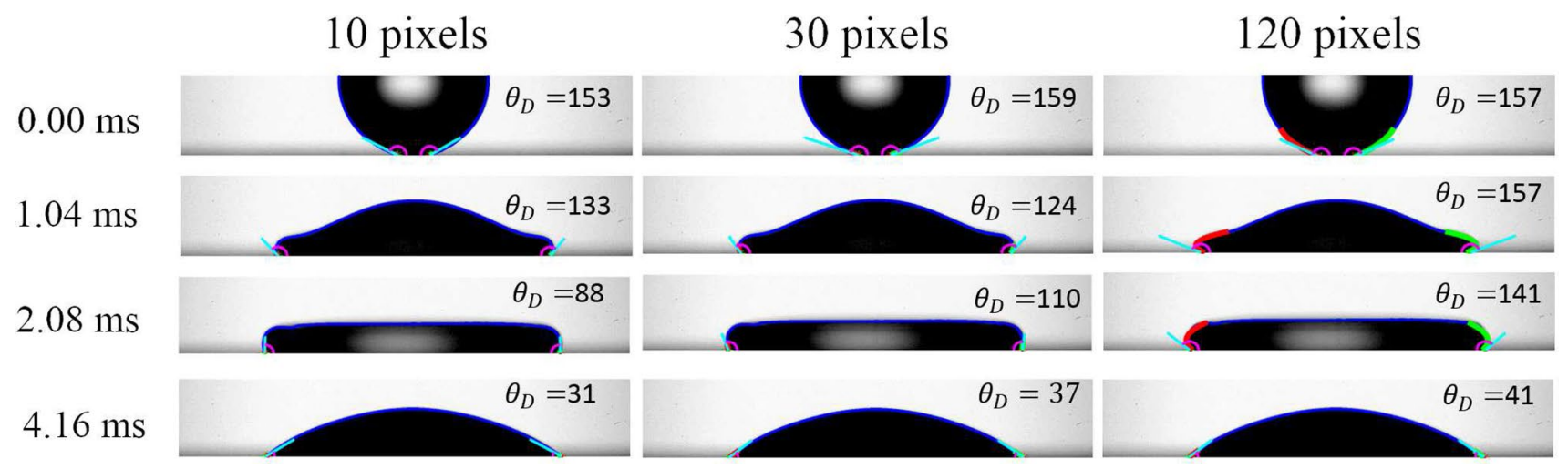

Alfonso A. Castrejón-Pita

alfonso.castrejon-pita@wadham.ox.ac.uk

J. Rafael Castrejón-Pita

castrejon@cantab.net

1 Department of Engineering Science, University of Oxford, Oxford OX1 3PJ, UK

2 School of Engineering and Materials Science, Queen Mary University of London, London E1 4NS, UK

\section{Introduction}

Quantifying the wettability of a liquid on a solid substrate is critically important for situations where either liquid adhesion or repellence is required. Industrial processes such as coating (Yarin 2006) and the spraying of pesticides (Bergeron et al. 2000) are examples where maximising the liquid adherence to a solid is desired. In contrast, repellence is sought in the design of materials with anti-icing properties (Liu et al. 2017) or impermeable clothing (Zhang et al. 
2018). How much a liquid wets a solid is known to depend on the properties of both, the liquid and the solid substrate, and is commonly studied through the measurement of the contact angle. This contact angle is defined as the geometric angle between the tangent of the droplet surface and the tangent to the solid surface at the triple point, i.e. the angle formed by the intersection of the liquid-solid and the liquid-vapour interfaces (Joanny and De Gennes 1984; Eral and Oh 2013). At the triple point, a solid, a liquid droplet and the surrounding gas are all in contact, creating three relevant surface effects, i.e. the solid-liquid $\gamma_{s l}$, solid-gas $\gamma_{s v}$ and liquid-gas $\gamma_{l v}$ forces. A sessile droplet on a solid surface adopts a semi-spherical shape reaching a minimum energy state and equilibrium (Chen 2013; Yuan and Lee 2013); this balance is described by the Young's equation. For a sessile droplet on an ideal flat surface that is smooth, homogeneous, rigid and insoluble, the contact angle is given by:

$\gamma_{s v}=\gamma_{s l}+\gamma_{l v} \cos (\theta)$,

where $\theta$ is the so-called Young's contact angle (Good 1992). Equation 1 represents the balance of surface tension forces between solid, liquid and gas (Bonn et al. 2009) and is satisfied when thermodynamic equilibrium is reached. At equilibrium, a unique equilibrium contact angle and diameter $D_{e q}$ are obtained regardless of the mechanism used to deposit the droplet on the substrate (Bonn et al. 2009). In practice, the angle given by Eq. 1 is not experimentally measurable, as substrate heterogeneities lead to thermodynamic metastable states, affecting the contact angle (Marmur 1994). In contrast, experiments determine the apparent contact angle, which is associated with the macroscopic geometry achieved by the liquid surface. In fact, every surface has at least two asymptotic possible values for the apparent contact angle: the advancing and receding angles $\left(\theta_{a}\right.$ and $\theta_{r}$, respectively). The former is seen when a droplet spreads over a solid surface, and the latter is observed on receding droplets (Yarin 2006). It has been argued that the $\theta_{a}$ and $\theta_{r}$ are the contact angles that truly describe substrate wettability (Huhtamäki et al. 2018). The difference between the maximum and the minimum contact angles is known as the contact angle hysteresis (Good 1992). Additionally, there are two categories of advancing and receding angles, namely dynamic and quasi-static. The dynamic advancing and receding angles are found when the contact line is in motion and far from equilibrium. In contrast, the quasi-static advancing and receding angles are found when the droplet is sessile or not largely deformed even if the contact line is moving (Yarin 2006; Snoeijer and Andreotti 2013).

The preferred technique to measure the quasi-static contact angles: $\theta_{a}$ and $\theta_{r}$, is the sessile-drop method that consists on pumping liquid into and out of a droplet resting on a substrate- measuring the advancing and receding angles, respectively (Eral and Oh 2013; Huhtamäki et al. 2018). The advantage of this method is that it also uses conventional optical imaging. The disadvantages are that is droplet size dependent and droplet shapes can be distorted by the wettability of the feeding needle (Good 1992; Eral and Oh 2013). However, in many other situations, where the contact line is far from equilibrium, quasi-static angles are not appropriate to characterise the surface wettability. Examples of where the contact line is far from equilibrium are found during the spreading or receding of an impacting droplet on a dry solid substrate. Many studies have been devoted to understanding these phenomena, finding that these dynamics are controlled by a subtle interplay between inertia, viscosity and capillary forces. The dynamics of drop impact onto solid substrates has received much attention due to their relevance in inkjet printing (Derby 2010), paint spraying (PasandidehFard et al. 2002) and other aerosol-based coatings (Fogliati et al. 2006). At least six different outcomes of drop impact have been identified: deposition, prompt splash, corona splash, receding break-up, partial rebound and complete rebound (Rioboo et al. 2001) (see Yarin 2006; Josserand and Thoroddsen 2016) for an extensive review). Upon drop impact, past works have shown that the contact diameter grows as $D \propto t^{1 / 2}$ (where $t$ is the time after impact) until it reaches a maximum value $D_{\max }$ (Yarin 2006). In this initial stage, droplets can slowly recede to acquire a smaller equilibrium contact diameter $D_{\mathrm{e} q}$. Under some conditions, a second spreading/receding phase can then be observed (Rioboo et al. 2001) that ends with the drop oscillating around the equilibrium contact diameter (Bayer and Megaridis 2006). The first spreading stage is commonly characterised in terms of the spread factor $d(t)=\frac{D(t)}{D_{0}}$, or the maximum spread factor $d_{m}=\frac{D_{\max }}{D_{0}}$, where $D_{0}$ is the diameter of the drop prior impact.

Past works have found $d_{m}$ to be in the range of 1.3 to 5.0 depending on the impacting conditions (Šikalo et al. 2002; Rioboo et al. 2002; Josserand and Thoroddsen 2016). In fact, for perfectly non-wetting substrates, Eggers et al. (2010) proposed the scaling $d_{m} \propto \operatorname{Re}^{1 / 5} f\left(W e R e^{-2 / 5}\right)$, where $d_{m}$ is found to be dependent on the interplay between viscous and capillary effects. Here, Re is the Reynolds number $\operatorname{Re}=\rho U_{0} D_{0} / \mu$ and We is the Weber number We $=\rho U_{0}^{2} D_{0} / \sigma$, where $U_{0}$ is the droplet impact velocity and $\mu, \rho$ and $\sigma$ are the fluid dynamic viscosity, density and surface tension, respectively. This scaling reduces to $d_{m} \propto W e^{1 / 2}$ for high impact speeds (i.e. for conditions where viscous effects can be neglected) and to $d_{m} \propto R e^{1 / 5}$ in the viscous regime (Eggers et al. 2010). This scaling has been experimentally validated by Laan et al. (2014). Other models have been proposed for wetting (dissipative) substrates (Šikalo et al. 2005; Bonn et al. 2009). According to Lee et al. (2016a, b) the impact dynamics can be determined either by the liquid characteristics and the 
substrate roughness or the dynamic contact angle $\left(\theta_{D}\right)$ at $d_{m}$. Moreover, Visser et al. (2015) conducted experiments with micrometer-sized droplets, concluding that the drop impact phenomena are scale invariant and dependent of both Re and We. In contrast, experimental studies by Šikalo et al. (2002) suggest that $d_{m}$ depends on $D_{0}$, as viscosity effects are dependent on the droplet size. Additionally, for drops impacting stainless steel, glass and paraffin, past results have found that $d_{m}$ depends on both the contact angle and a critical Weber number (Šikalo et al. 2005; Vadillo et al. 2009; Lunkad et al. 2007; Chen 2013).

Furthermore, Yokoi et al. (2009) ran numerical simulations of droplets spreading over solid substrates using different contact angle models: dynamic, equilibrium and static contact angles. Numerical results were compared with experiments carried out by Vadillo et al. (2009) concluding that the dynamic contact angle model produced the best agreement with experiments. Likewise, de Goede et al. (2019) concluded that, for impact velocities $U_{0}<1$ $\mathrm{m} / \mathrm{s}$, surface wettability can be used to predict $d_{m}$. Moreover, Quetzeri-Santiago et al. (2019b) demonstrated that the dynamic contact angle parametrises the splashing outcome of impacting droplets on solid wettable and non-wettable substrates.

As discussed above, it is clear that many past research on droplet spreading and splashing relies on the detection of the contact line and on the measurement of the contact angle. However, a standard measurement method is unavailable and, consequently, the contact angle is often obtained and reported using different techniques. One of the most widely used practices to obtain the equilibrium contact angle is through the axisymmetric drop shape profiling. In this method, the contact angle is measured by numerically fitting a Laplace equation to the droplet surface profile (Rotenberg et al. 1983; Del Rio and Neumann 1997). This method requires perfectly axisymmetric and sessile droplets (Bateni et al. 2003; Del Rio and Neumann 1997). An alternative method is to approximate the droplet profile to a sphere or an ellipse (Lamour et al. 2010) and obtain the contact angle from these fittings. Importantly, these two methods are not suitable for conditions where a droplet is largely deformed or cannot be modelled by a spherical cap, e.g. during droplet impact. In these cases, the goniometric mask method is often used. In this approach, a goniometer is digitally located at the contact line to automatically measure the contact angle formed at its proximity by the droplet (Biolè and Bertola 2015; Lee et al. 2016a, b). Another suitable method for measuring the contact angle is through the fitting of a polynomial function to the droplet profile. This method provides accurate results at a minimal computational cost (Chini and Amirfazli 2011; Bateni et al. 2003; Chen et al. 2018). In fact, Bateni et al. (2003) and Atefi et al. (2013) studied the effect of the fitting polynomial order on the measurement of the contact angle for sessile droplets finding that, with the suitable parameters, results reproduce those of the axisymmetric drop shape analysis profile (ADSA-P).

These last two works are the exception to the norm, as many other past researches do not describe in detail the methods used to determine the contact angle and often adopt imaging processing algorithms without further testing their reliability. Most past works have focused on measurements of the contact angle of sessile or quasi-static droplets and not on the contact angle of rapidly moving contact lines. Furthermore, the vast majority of these previous studies do not report on the details of error analysis of uncertainty sources. In this paper, we use various polynomial fittings to measure the dynamic contact angle, i.e. the rapidly advancing and receding contact angles of an impacting droplet on a solid substrate. We contrast their results and discuss their differences. Additionally, we analyse the effect of an inadequate detection of the contact line (pinning points) on the measurement of the contact angle. In particular, we focus on millimeter-sized drops impacting onto hydrophilic substrates $(15<\theta<90)$, with impact velocities leading to spreading and simple deposition $\left(1.1<U_{0}<2.1 \mathrm{~m} / \mathrm{s}\right)$. The aim of the paper is to highlight the difficulties encountered when measuring the dynamic contact angle, the contact line diameter and the contact line velocity.

\section{Experimental method}

The experiments consist of visualising single drops, impacting dry solid substrates. In our conditions, the substrate is perpendicular to the impact direction. Three substrates were used: glass, Teflon-covered glass and acrylic. Water-glycerol solutions and pure water drops (see Table 1 for fluid characteristics) were produced by two methods: by dripping and by using a drop-on-demand (DoD) generator. In the former, a pump slowly pushes the liquid at the end of a syringe tip (of $1.0 \mathrm{~mm}$ diameter) until a drop falls. In the latter, an electromagnetic actuator pushes the liquid through a nozzle to create a droplet (Castrejón-Pita et al. 2008). In the DoD experiments, a $1.0 \mathrm{~mm}$ outer diameter conical nozzle was used and the driving signal is a single square pulse. In our experiments, drop impact speeds ranged from 1.1 to $1.7 \mathrm{~m} / \mathrm{s}$, and the drop diameter ranged from 1.1 to

Table 1 Fluid properties used throughout the experiments

\begin{tabular}{llll}
\hline Fluid & $\begin{array}{l}\text { Viscosity }(\mathrm{mPa} \\
\mathrm{s})\end{array}$ & $\begin{array}{l}\text { Surface tension } \\
(\mathrm{mN} / \mathrm{m})\end{array}$ & Density $\left(\mathrm{kg} / \mathrm{m}^{3}\right)$ \\
\hline Water & 0.9 & 70.8 & 998 \\
Sol 1 & 2.0 & 70.3 & 1065 \\
Sol 2 & 60.0 & 66.0 & 1126 \\
\hline
\end{tabular}


$2.5 \mathrm{~mm}$. Table 2 summarises our experimental conditions. The impact events were captured using a Phantom V710 coupled to a $12 \times$ Navitar microscope lens in a shadowgraph configuration. The camera resolution was set to $1280 \times 256$ pixels ${ }^{2}$ with a sample rate of 23,000 frames per second with an exposure time of $10 \mu \mathrm{s}$. We studied the magnification effect on the algorithm method using three different effective resolutions of the experiments: $3.91 \mu \mathrm{m}, 6.47 \mu \mathrm{m}$ and $8.86 \mu \mathrm{m}$ per pixel. The camera is inclined $\approx 2^{\circ}$ to obtain a clear image of the contact line; the effect of this inclination on the measurement of the contact angle is negligible and discussed in Sect. 3.2 (Quetzeri-Santiago et al. 2019a). Moreover, we ensured that the droplet was perfectly focused and the aperture/iris was (for our light settings) remained as closed as possible to maximise the depth of field. A 300 W LED light source and an optical diffuser were utilised to produce a uniform bright backlight.

In this investigation, we use a MATLAB routine to measure the contact angle. The code works by fitting a polynomial of order $m$ to a section of the droplet profile near the contact line. The droplet and substrate profiles are obtained by using a defined intensity threshold for the conversion of a greyscale image to a binary format, using Otsu's method (Otsu 1979). This method automatically chooses the threshold value that minimises the intraclass variance of the thresholded black and white pixels. The image processing steps are shown in Fig. 1. The droplet and substrate profiles are then extracted as an array of pixels. The code first detects the substrate "horizon" by identifying the first and last profile pixels of the binary image. Any pixel structure above this horizon is considered part of the droplet, where the first out-of-line pixels from each side are identified as the contact points. The position of these points is recorded in all the images to track the spreading diameter and thus the contact line velocity. The left-hand and right-hand sides of the droplet boundary are independently analysed. For each side, the code selects a set region of the droplet boundary array starting from the pinning point to a set perimeter length $\delta_{n}$. This region forms an interrogation area defined by the length $\delta_{n}$, as illustrated in Fig. 2a. The code then fits an $m$-order polynomial function with the least squares method (OLS) to the $n$ pixels of the boundary ( $\delta_{n}$ in each side). Finally, the algorithm computes the fitting derivative and
Table 2 Experimental conditions

\begin{tabular}{llllllc}
\hline $\begin{array}{l}\text { Experiment } \\
\text { number }\end{array}$ & $\begin{array}{l}\text { Drop generation } \\
\text { method }\end{array}$ & Substrate & Liquid & $D_{0}(\mathrm{~mm})$ & We & Re \\
\hline 1 & Dripping & Acrylic & Water & 2.41 & 43 & 2736 \\
2 & DoD & Acrylic & Water & 2.47 & 49 & 2964 \\
3 & DoD & Acrylic & Water & 1.12 & 45 & 1904 \\
4 & DoD & Acrylic & Sol 1 & 2.40 & 166 & 2717 \\
5 & DoD & Acrylic & Sol 2 & 2.10 & 169 & 870 \\
6 & Dripping & Glass & Water & 2.53 & 46 & 2884 \\
7 & DoD & Glass & Water & 2.63 & 46 & 2959 \\
8 & DoD & Glass & Water & 1.10 & 44 & 1887 \\
9 & DoD & Glass & Sol 1 & 2.42 & 162 & 2662 \\
10 & DoD & Glass & Sol 2 & 1.98 & 164 & 832 \\
\hline
\end{tabular}

(a)

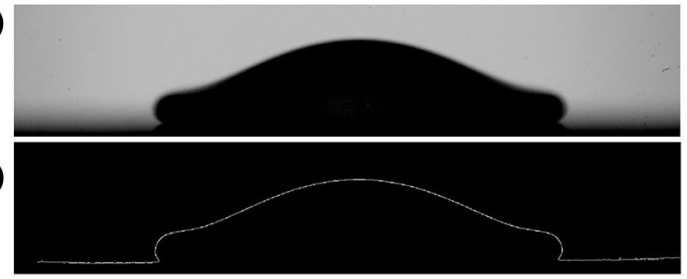

(b)

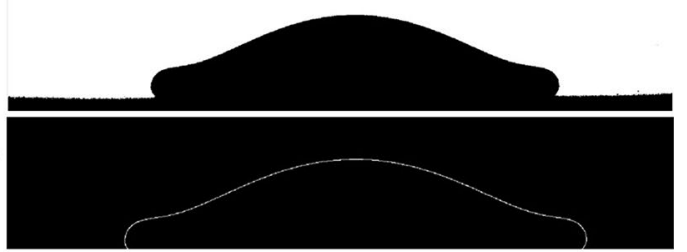

(e)

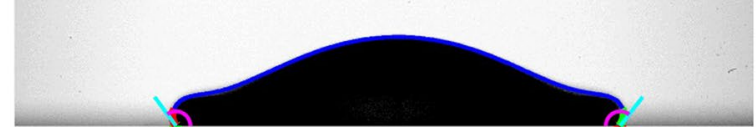

Fig. 1 Image processing steps: a original image, $\mathbf{b}$ grey scale image converted to binary image using Otsu's method, c detection of the boundary of the droplet and substrate, d boundary of the droplet without the substrate and e original image after the processing. The navy blue contour corresponds to the droplet boundary, the green (left) and red (right) stars show the pinning points, the light blue lines are the tangent evaluated at the pinning point and the pink arcs correspond to the contact angle 


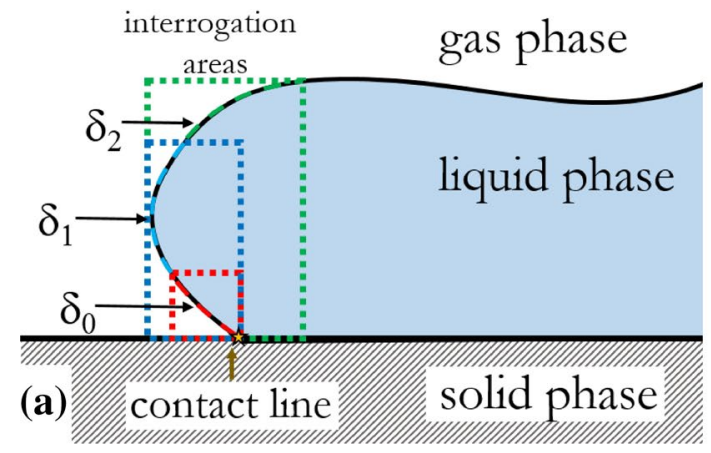

Fig. 2 Sketch showing the variables studied in this work. The contact line (or the triple point) is shown as a star and indicates the place where all the three phases meet. In $\mathbf{a}$, the interrogation areas define a perimeter along the droplet's profile of size $\delta$ (in pixels). In $\mathbf{b}$, the

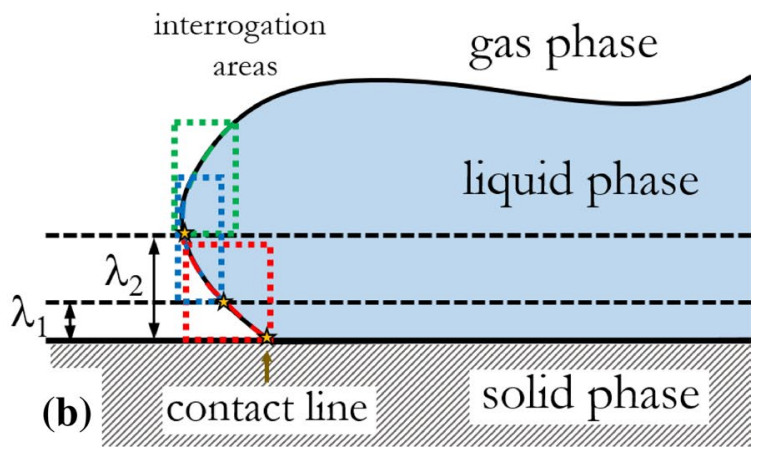

substrate horizon is illustrated as a black thick line. In practice, this horizon might be misplaced by image analysis algorithms by a height $\lambda$ due to the interface being out of focus or fuzzy

an offset $\lambda_{n}$ on the detection of the substrate position on the measurement of the contact angle as illustrated in Fig. 2b. Our results are discussed in the following sections where we also describe the most reliable conditions to measure the dynamic contact angle.
In this manuscript, we analyse the value of the contact angle as a function of $\delta_{n}$, i.e. the number of pixels $n$ along the droplet profile used to fit the polynomial, and the order

$m$ of the polynomial. Additionally, we study the effect of

evaluates it at the pinning point: the contact angle is then computed from this value. For simplicity, we chose the interface points at the centre of the pixels (no sub-pixelar resolution). (a)

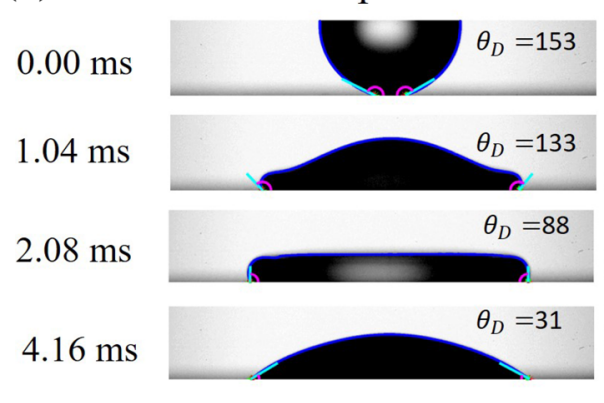

(b)

$0.00 \mathrm{~ms}$

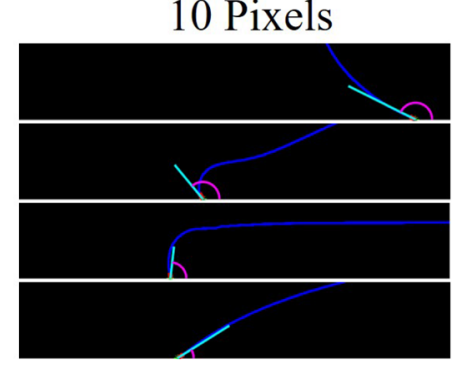

$1.04 \mathrm{~ms}$

$2.08 \mathrm{~ms}$

$4.16 \mathrm{~ms}$
30 pixels
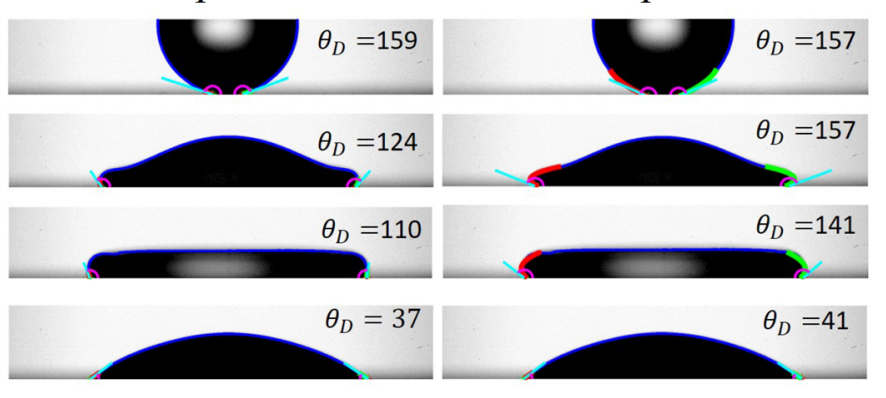

30 Pixels
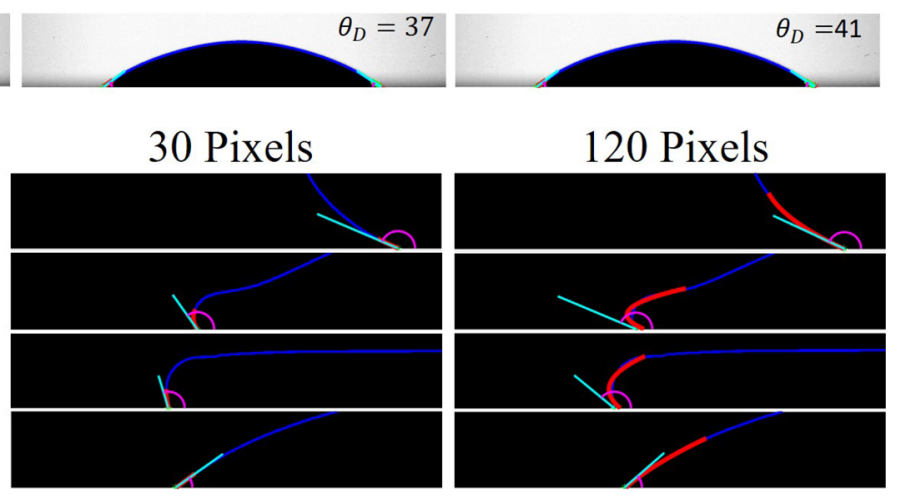

\section{Pixels}

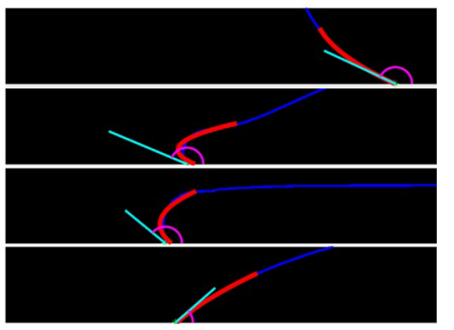

Fig. 3 a Example snapshots of the experimental and analysed images. The three sets correspond to the MATLAB processed images of a drop in the spreading phase. The images are arranged according to the number of pixels used to fit a second-order polynomial to calculate the contact angle, i.e. 10 pixels $\rightarrow \delta_{1} / D_{0}=0.0301,30$ pixels $\rightarrow \delta_{2} / D_{0}=0.092$ and 120 pixels $\rightarrow \delta_{3} / D_{0}=0.369$. The navy blue contour corresponds to the droplet boundary, the red (left) and green (right) stars show the pinning points, the light blue lines correspond to the tangent evaluated at the pinning point and the pink arcs correspond to the contact angle; $\mathbf{b}$ shows a close-up of the droplet contour detected by the MATLAB algorithm 


\section{Results and discussion}

Examples of our experimental results and analysis are shown in Fig. 3 where a sequence of images of a water drop impacts an acrylic substrate. The first spreading and receding phases are observed at various times after impact. In the hydrophilic surfaces studied here, receding is negligible. In this work, we have focused our analysis on four different instants, i.e. (i) the time of the first contact ( $t=0.00 \mathrm{~ms}$ in Fig. 3), (ii) the point where $d(t)=d_{m} / 2(t=1.04 \mathrm{~ms}$ in Fig. 3), (iii) the time at maximum spreading $d(t)=d_{m}(t=2.08 \mathrm{~ms}$ in Fig. 3) and (iv) the first point of receding ( $t=4.16 \mathrm{~ms}$ in Fig. 3). These times show the most representative behaviours found during the dynamics of impact. In particular, at $d_{m} / 2$ the droplet is rapidly spreading and is highly deformed, a situation rarely analysed in the literature. Accordingly, at these times, the droplet contact angle is reported in terms of the droplet profile lengths $\left(\delta_{n}\right)$ and for various polynomial orders $m$. In addition, we also study the effect of an inaccurate detection
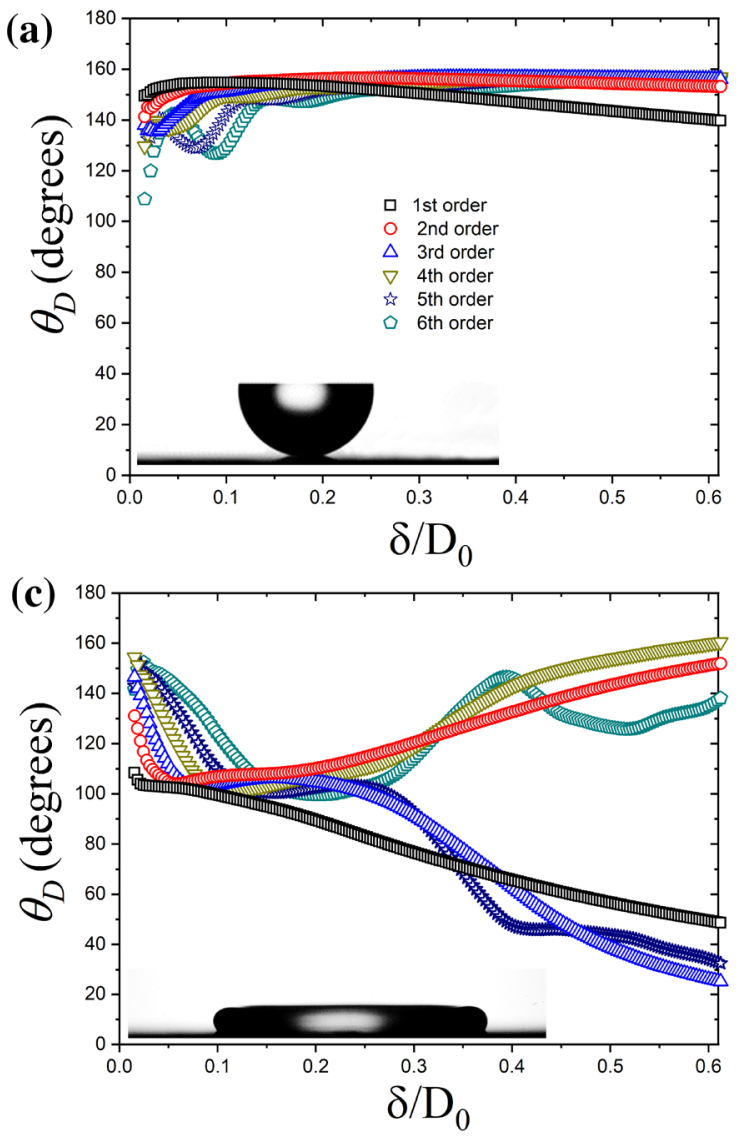

Fig. 4 Contact angle in terms of the number of pixels used to fit the droplet profile. Here, four instants found during droplet impact are analysed: a the time of first contact, $\mathbf{b}$ the point where $d(t)=d_{m} / 2, \mathbf{c}$ of the substrate position on the measurement of the contact angle.

\subsection{The polynomial fitting}

As noted by Bateni et al. (2003), the order of the polynomial used to adjust the droplet shape at the pinning point is crucial to the value of the contact angle on sessile droplets. In this work, we have extended this study to other conditions where the droplet is far from equilibrium and, thus, its shape differs from a spherical cap. Our first set of results is seen in Fig. 4 where the contact angle is obtained for various polynomial orders at the four relevant times previously discussed. Additionally, Fig. 4 shows the contact angle obtained in terms of the ratio between the number of pixels used to fit a $m$-degree polynomial and the diameter of the impacting droplet $\left(\delta / D_{0}\right)$. These results show that the measurement of the contact angle is sensitive not only to the polynomial order but also to the instantaneous shape of the droplet. In fact, for highly deformed droplets, differences of up to $100^{\circ}$ in the measured contact angle are seen between
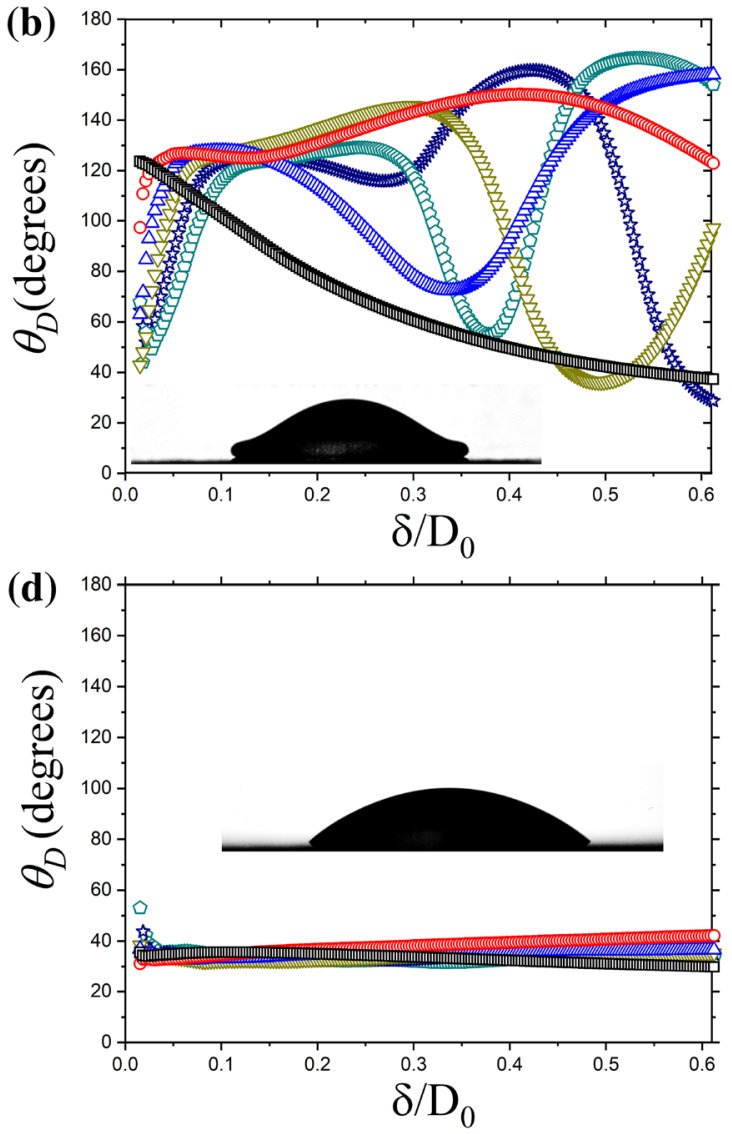

the time at maximum spreading diameter, $d_{m}$ and $\mathbf{d}$ the time when the receding phase starts. The contact angle obtained from six different polynomial fittings for Experiment 5 (Table 2) 
the different-order polynomials. In these conditions, the polynomial order showing the largest differences is that corresponding to a linear fit. As seen, for the linear fit $(m=1)$, the dynamic contact angle decreases monotonically for increasing profile lengths for the four droplet shapes studied here. In fact, even at instants where the droplet resembles spherical shapes, i.e. first contact (Fig. 4a) and first receding instants (Fig. 4d), differences on the contact angle of up to $30^{\circ}$ are found for $m=1$. In contrast, higher-order polynomials reach a stable contact angle value as the size ratio domain is increased.

Largely deformed droplets also offer intricate variations (Fig. 4b, c). Here, large differences in the contact angle value are observed for the various polynomial fittings and fitting domains. As seen in our results, variations of up to $80^{\circ}$ can be obtained for droplets shapes in the early spreading phases (Fig. 4b) or up to $30^{\circ}$ at the maximum spreading diameter where droplets acquire the characteristic pancake shape (Fig. 4c). In practical terms, the contact angle should be measured at the proximity of the contact line; consequently, any measuring method should include an upper limit for the length domain. Moreover, as seen in Fig. 5, for a time at $d(t)=d_{m} / 2$ a large number of fitting pixels translate in an inadequate fitting of the droplet profile. This is due to the high droplet deformation far from the contact line. Additionally, a lower domain limit should also exist for the fitting to correctly represent the droplet shape. Without a standard reference, or a theoretical value for the apparent dynamic contact angle to compare our results with, we quantify the differences from the various fitting domains through the standard deviation for different effective optical resolutions. Figure 6 shows the standard deviation in terms of the parameter $\delta / D_{0}$ for different image resolutions and from the various polynomials used in this paper, for each $\delta / D_{0}$. Three resolutions are used: 3.91 (black squares), 6.47 (cyan circles) and 8.89 (blue triangles) $\mu \mathrm{m} /$ pixels.

As expected, the standard deviation obtained from all the polynomials is consistently low for the receding case where the shape resembles a spherical cap. An exception is the data set with an effective resolution of $8.86 \mu \mathrm{m} /$ pixel and for a domain larger than $\delta / D_{0}=0.25$. We attribute this to an overfitting of the droplet profile. In this condition, any domain larger than $10 \%$ of the droplet diameter produces a standard deviation of less than $5^{\circ}$. A similar behaviour is found at the point of first contact where a low deviation is seen for domains larger than $30 \%$ of the droplet diameter. As discussed, in these two cases, the droplets are not largely deformed, resemble spherical bodies, and, therefore, good fittings are obtained over a large fitting domain. The standard deviation for largely deformed cases is rich but shows limited variations at short profile domains. Across all our experiments, the largest error is seen for the $3.91 \mu \mathrm{m} / \mathrm{pixel}$ resolution, which arises from the blurring for the contact point. In fact, this effect is coupled to the lens settings and characteristics as for our long distance microscope lens, an increase in the magnification reduces the depth of field. Furthermore, two lenses working at the same magnification can have different depth of fields, according to their aperture, and consequently produce measurements with different standard deviations. Importantly, the standard deviations present local minima around the domain range of $\delta / D_{0}=$ 0.04 to 0.10 (or 13 to 32 pixels for an effective resolution of $6.47 \mu \mathrm{m} / \mathrm{pixel}$ ), where the various polynomial fittings seem to agree within a standard deviation of $15^{\circ}$. Moreover, the standard deviation in this region is particularly low $\left(\approx 5^{\circ}\right)$ for the highly deformed shapes and the receding case. As a result, we conclude that a robust domain for a polynomial fitting is within the range corresponding to profile lengths of 4 to $10 \%$ of the droplet diameter, for the three effective resolutions used in this study. This fitting range is consistent with previous research asserting that larger domains might not trace the drop profile accurately (Chini and Amirfazli 2011; Andersen and Taboryski 2017). This upper limit has also been discussed by Biolè and Bertola (2015), where their domain is determined by the need of their mask to follow the droplet curvature, which requires a small mask, and "accuracy in their area measurement, which requires a bigger mask". Technically speaking, our upper domain

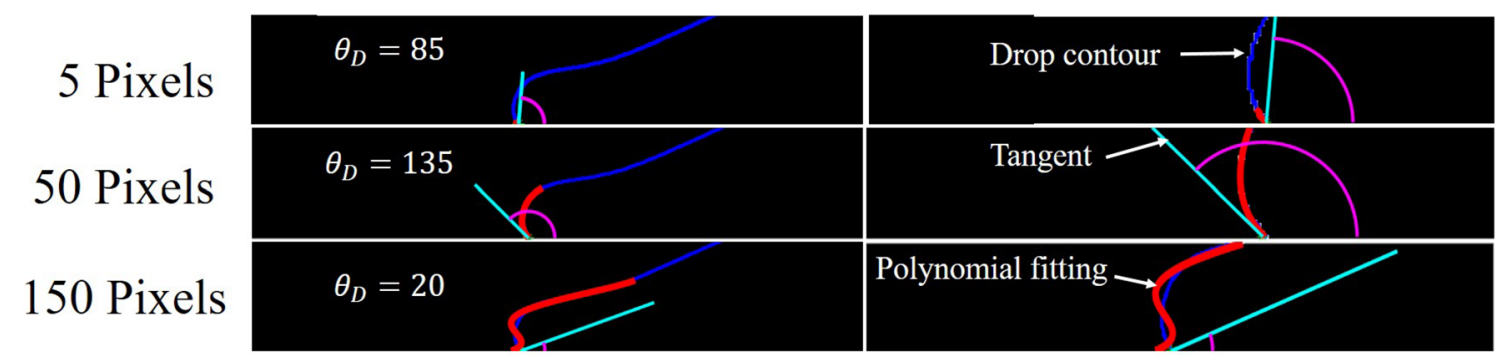

Fig. 5 Image analysis results of a fourth-order polynomial fit for various number of adjusted pixels $\delta / D_{0}$, at a time when $d(t)=d_{m} / 2$. This example shows that the polynomial no longer faithfully represents the profile of the droplet. This is due to the high droplet deformation far from the contact line 

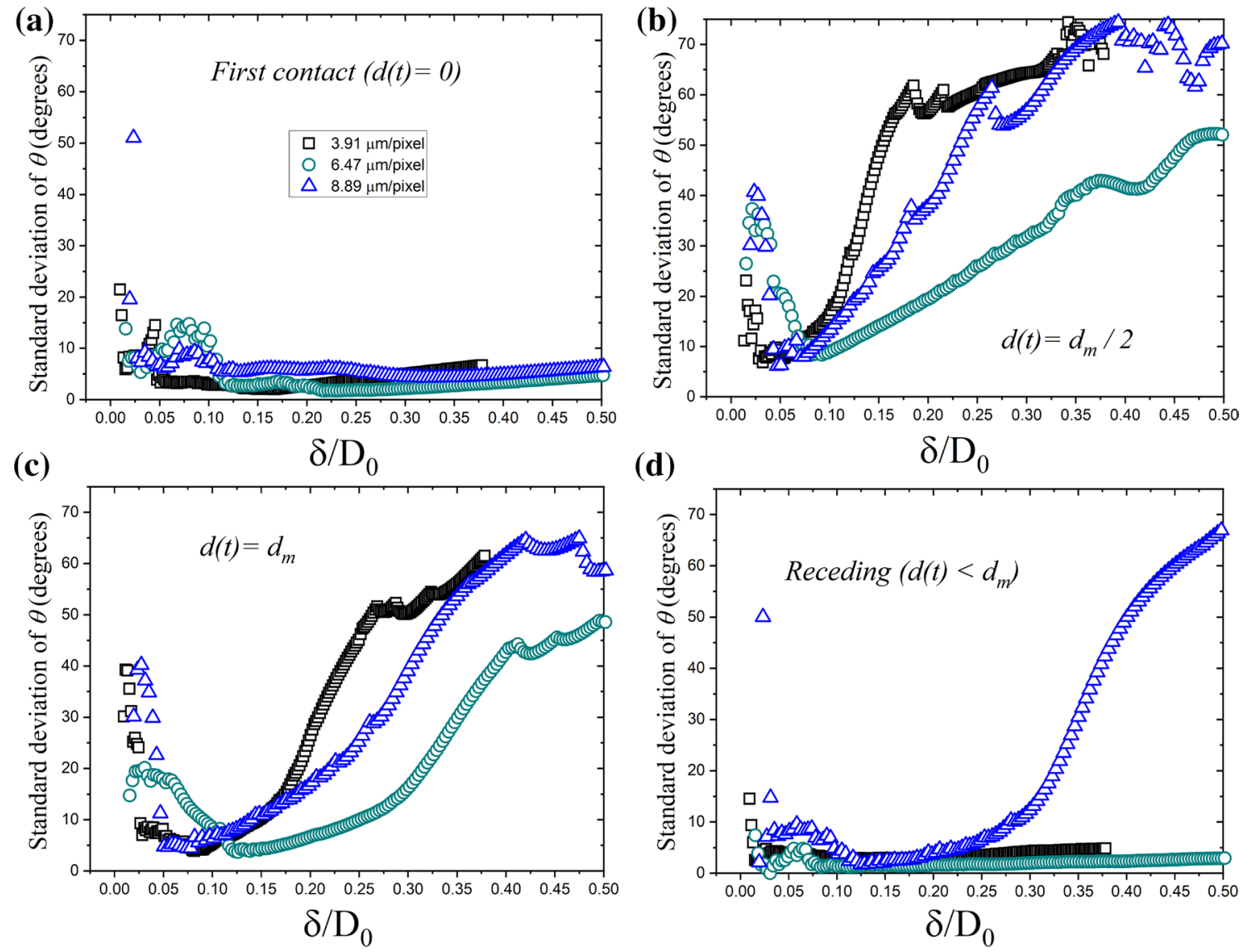

Fig. 6 Standard deviation in terms of the parameter $\delta / D_{0}$ for different image resolutions. The standard deviation is calculated based on all the polynomials used in the analysis, for each $\delta / D_{0}$. Black squares, cyan circles and blue triangles represent the various resolu-

tion used here, i.e. $3.91 \mu \mathrm{m} / \mathrm{pixel}, 6.47 \mu \mathrm{m} /$ pixel and $8.89 \mu \mathrm{m} / \mathrm{pixel}$, respectively. a Results at the time of first contact, $\mathbf{b}$ the time where $d(t)=d_{m} / 2$, c the time at maximum spreading $d(t)=d_{m}$ and $\mathbf{d}$ the time when the receding phase starts

Fig. 7 Standard deviation of the contact angle calculated in terms of the polynomial order. The standard deviation is associated with all the $\delta / D_{0}$ considered in this paper for each polynomial. As seen, the quadratic polynomial shows the smallest deviation for all cases

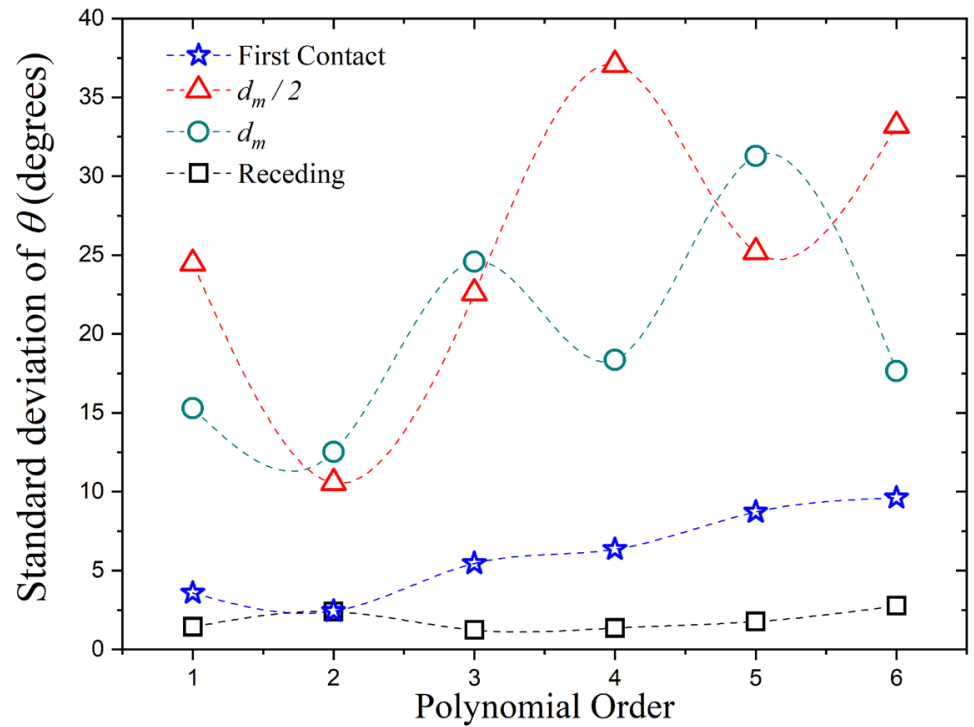


limit can extend to the contact line found at the other end of the droplet contour. However, as noted by other authors, we argue that the contact angle should be measured locally. Our optimum region of measurement is found where the standard deviation of the data is at its minimum value, across the various shapes.

Our next analysis focuses on the standard deviation in terms of the polynomial order; this is shown in Fig. 7. Interestingly, for the four shapes, the second-order polynomial fitting consistently produces the lowest standard deviation. In fact, this polynomial order has been used by other authors (Chini and Amirfazli 2011); here, we confirm that this fitting is the most robust for dynamic contact angle measurements.

Based on these results, we conclude that the fitting parameters that produce the most consistent results across conditions are a second-order polynomial fitting applied to a droplet profile length corresponding to $5 \%$ of that of the initial droplet diameter. Our standard deviation for these conditions is between $3^{\circ}$ and $5^{\circ}$ (Quetzeri-Santiago et al. 2019a, b). While this might appear large when compared against measurements of static contact angle measurements (where hundreds of measurements can be averaged for a single case), measurement of rapidly evolving contact lines is scarce, with authors usually not reporting errors (or simply assigning an ambiguous error). For instance, when using our algorithm to extract the static contact angle of a sessile drop for which 100 images exist (extracted from a highspeed movie) under our experimental conditions, the experimental error is $0.6^{\circ}$. In comparison, for the dynamic case, Lee et al. (2016b) reported errors on the dynamic contact angle in the range of $2.5^{\circ}$ to $9.8^{\circ}$ and a maximum difference of $5 \%$ between the polynomial fit method and the goniometric mask method. Therefore, in the following sections, we strictly use these conditions when reporting the contact angle.

\subsection{Contact line and pinning points}

In our experiments, special care was taken when setting the alignment and depth of field of our optical system to obtain a sharp and well-defined substrate boundary. In practice, observing a sharp boundary is often difficult and limited by the commercial availability of optical and illumination components, especially for sub-millimetre droplets. In this section, we evaluate the sensitivity of contact angle measurements on the correct identification of the location of the pinning points. As described above, this issue might arise on experimental set-ups where images are out of focus, blurred or short of the depth of field. In our experimental set-up, we can identify the true pinning points within two pixels but that might not be the case in other set-ups due to the conditions described above. Here, we study the effect of offsetting the position of the substrate plane (otherwise called horizon) on the measurement of the contact angle, mimicking potential visualisation limitations. This is illustrated in Fig. $2 b$, where we define an offset distance $\lambda$ (in pixels) that can be added in our algorithm to the "true" substrate position; $\lambda$ can be negative if the fitting is forced to commence below the true position or positive if above.

Figure 8 shows the variation of the contact angle value in terms of $\lambda$ for the four different droplet shapes (the domain shown here corresponds to $\lambda$ in the range of -10 pixels to 10 pixels). Our results are conclusive; an offset from the true pinning point can result in important differences in the measurement of the contact angle. In fact, an offset of only five pixels $\left(\lambda / D_{0}=0.02\right)$ is enough to produce differences of up to $19^{\circ}$ in the measurement. This miscalculation might be evident and easy to fix on a single picture, but dynamic systems require the automatic measurement of the contact angle for thousands of pictures where a plethora of shapes are found. The effect, during the spreading phase (Fig. 8b), leads to a difference of $24^{\circ}$ in the measured contact angle for a pinning point that is placed 10 pixels above its true position. Here, as done in other works, to avoid uncertainty and sharply capture the contact line, we inclined the camera into a small angle of approximately $2^{\circ}$. Inclining the camera, an angle $\phi$ affects the measurement of $\theta_{D}$ by altering the projected height $\left(h^{\prime}\right)$. The droplet height and the true height $h$ are related by $h^{\prime}=h \cos (\phi)$. Consequently, in our experiments, the angle of the camera only affects the measurement of the contact angle by $0.6 \%$, which is considered negligible in this work.

We conclude this section by noting that the contact line position should be identified within a distance that is less than $1 \%$ of that of the droplet in order to achieve reliable measurements of the contact line.

\subsection{Validation}

Following our reliability analysis, we used our experiments and technique to test some published results. The fitting conditions detailed above were used to study the spreading diameter in terms of the Weber number, which was varied by adjusting the impact speed. Figure 9 summarises the results. Here, the spreading diameters $d(t)$ are presented in terms of the dimensionless time $t^{*}=t U_{0} / D_{0}$ (from the time of impact). At early times $t^{*}<0.1 \mathrm{~ms}$, for hydrophilic substrates, our experiments indicate that substrate wettability does not significantly affect droplet spreading. As observed by Rioboo et al. (2002), surface wettability only comes into play at later times $\left(t^{*}>0.5\right)$. In addition, we observe that both the We and Re numbers affect the maximum spreading diameter $d_{m}$. Indeed, Experiments 4 and 9 have the same We number as Experiments 5 and 10, but Re numbers are approximately four times larger. Our results show that these 
(a)

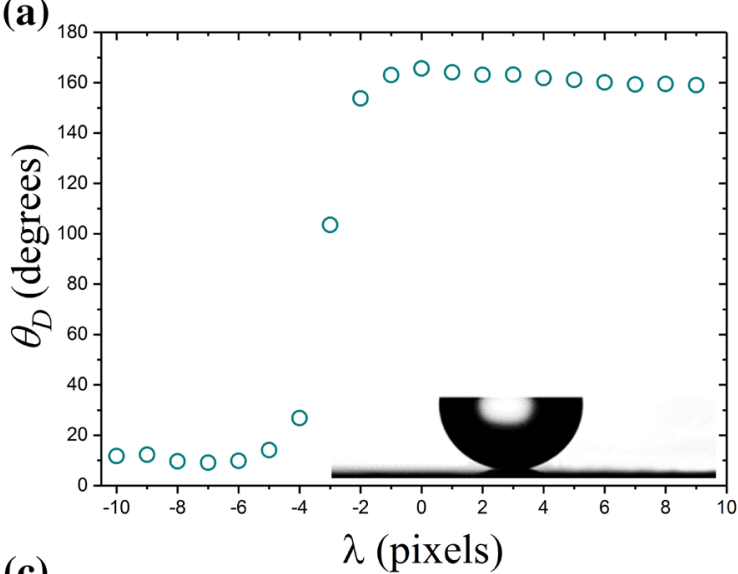

(c)

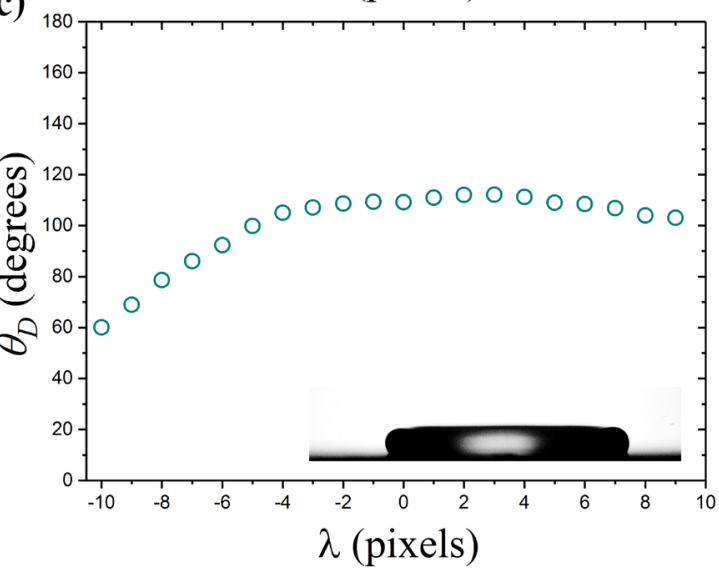

(b)

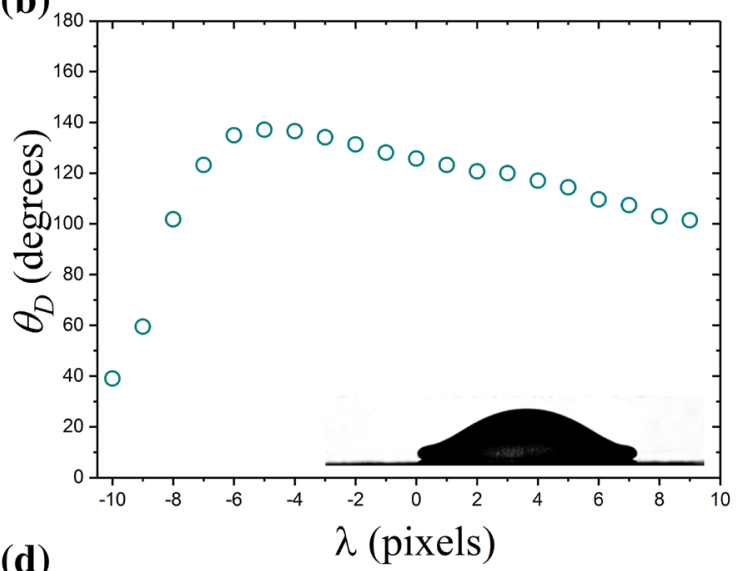

(d)

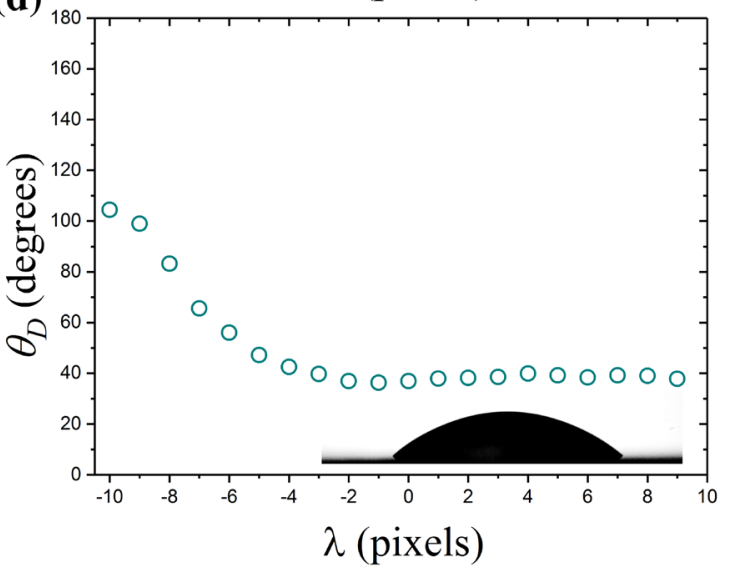

Fig. 8 Influence of a vertical offset applied to the contact line (of height $\lambda$ ) on the contact angle measurement. The offset is set manually within the MATLAB code, from 10 pixels below to 10 pixels

above the contact line. As seen, the measurement of the contact angle is critically dependent on the correct detection of the contact line

(b)

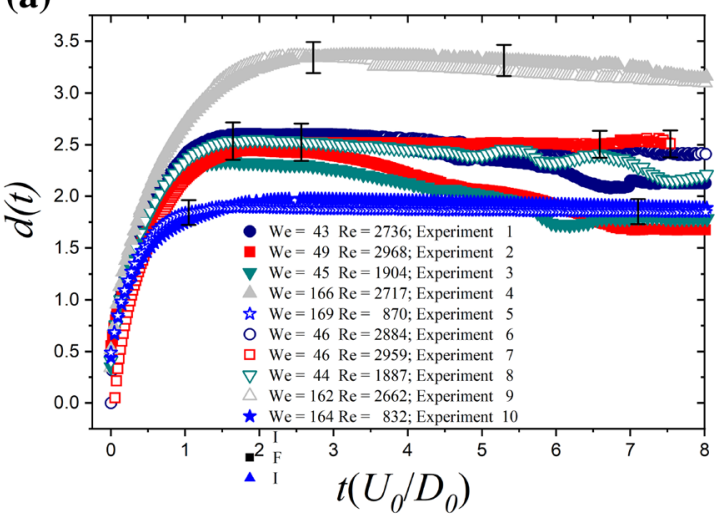

Fig. 9 a Contact diameter $d(t)$ in terms of the dimensionless time. b Contact angle in terms of the contact line velocity. For both graphs, hollow symbols represent experiments done on glass and solid symbols on acrylic

differences in Re numbers produce dramatic changes in the dynamics, finding that $d_{m}$ doubles for the high Re number cases. This difference is also reflected at the contact angle dynamics (Fig. 9b). Furthermore, we note that there are discrepancies in the results from the dripping and the dropon-demand generation methods that have similar We and Re numbers (Experiments 1 and 2, and 6 and 7). These discrepancies are due to differences in the on-flight oscillatory 
behaviour between droplets generated by DoD or dripping. These differences induce various oscillatory modes (and/or phases, if they both oscillate at the fundamental mode). It is well known, for instance, that these oscillations change the "effective" radius of curvature of the front of the drop, leading to radically different results during splashing (Thoraval et al. 2013)

Figure $9 \mathrm{~b}$ shows the contact angle in terms of the contact line velocity $u_{c l}$. As described in previous articles, at the first instants after impact, and near the contact point, the drop is greatly deformed and the contact angle is approximately $180^{\circ}$, while the top of the droplet remains spherical. At these early times, the contact line velocity can move at up to $u_{c l}=15 \mathrm{~m} / \mathrm{s}$, decreasing rapidly as the contact angle goes from $180^{\circ}$ to a local minimum. Subsequently, as seen in the insert of Fig. 9b, the contact angle value reaches a long-lived asymptotic contact angle $\left(\theta_{D} A\right)$ at a contact line velocity range between 0.5 and $3.0 \mathrm{~m} / \mathrm{s}$ (Quetzeri-Santiago et al. 2019b). Eventually, the contact angle reaches its equilibrium value within the hysteresis at $u_{c l}=0.0 \mathrm{~m} / \mathrm{s}$.

High-viscosity droplets, Experiments 5 and 10 in Fig. 9, corresponding to $\mu=60 \mathrm{mPa}$ s show substantially different dynamics at low spreading speeds, where a clear local maximum value is visible at $u_{c l} \approx 0.5 \mathrm{~m} / \mathrm{s}$. This is consistent with the results by Vadillo et al. (2009), where an asymptotic contact angle was observed for low-viscosity fluids and a large hysteresis for high viscosity liquids. As highlighted previously, the dynamic contact angle is the result of the interplay between inertia, capillary and viscous forces (Vadillo et al. 2009; Yokoi et al. 2009) where greater capillary and viscous forces imply larger contact angles. This is well reflected in our results, where hydrophilic substrates, at a given We number, are associated with large hysteresis and short spreading (small $d_{m}$ ). This is in agreement with previous results where $d_{m}$ has been found to be dependent of the dynamic contact angle $d_{m}$ (Vadillo et al. 2009; Lee et al. 2016a, b).

As mentioned in the introduction and in past works, the contact angle is affected by the substrate properties and the energy dissipation at the contact line. Therefore, following the approach of Lee et al. (2016a, b), we use the moving contact angle to parametrise the maximum spreading diameter. As argued in previous papers, $\theta_{D A}$ contains information about the interplay between viscous and capillary forces acting on the contact line (Šikalo et al. 2005). Moreover, the scaling $d_{m} \sim W e^{1 / 4}$ was found to fit the data for water (Laan et al. 2014). Combining these two findings, we propose a scaling of the form $d_{m} \sim W e^{1 / 4} f \theta_{D A}$. Furthermore, our experiments indicate that the larger the viscosity, the larger the $\theta_{D A}$, and the smaller the $d_{m}$. In fact, our results indicate that the scaling $d_{m} \sim W e^{1 / 4}$ alone overpredicts $d_{m}$ for viscous droplets. In contrast, for $\theta_{D A}<90^{\circ}$, the scaling underpredicts $d_{m}$. Therefore, we propose a factor $f\left(\theta_{D A}\right)=1 \pm \cos ^{2}\left(\theta_{D A}\right)$

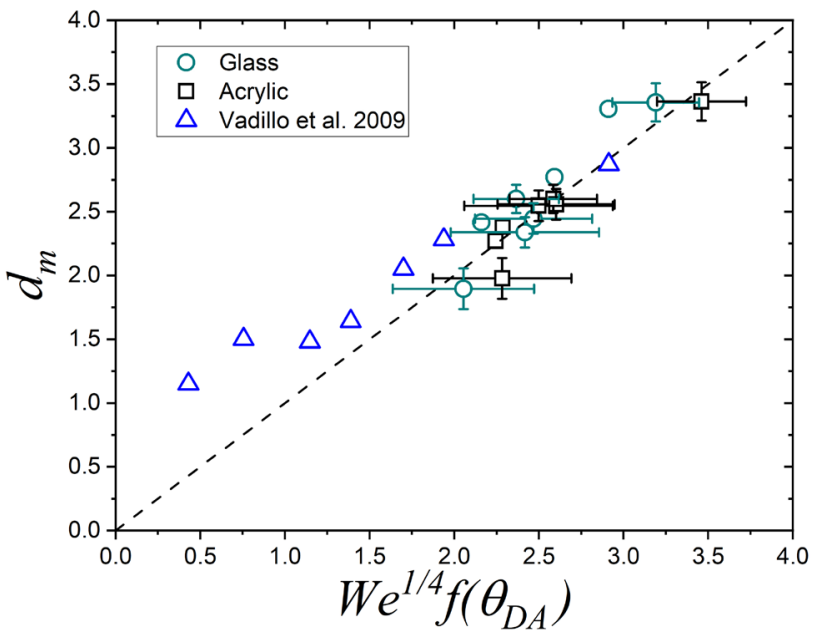

Fig. 10 Comparison between the experimentally obtained maximum spreading factor (vertical) and that obtained from Eq. 2 (horizontal axis). The error bars take into account the associated errors on the measurement of the contact angle, the impact speed, droplet diameter, density and surface tension. Additionally, we have included data from Vadillo et al. (2009)

in addition to the $W e^{1 / 4}$ scaling. Here, $\theta_{D A}$ is obtained by averaging the dynamic contact angle measurements over the range of $0.25<\mathrm{ucl}<3.0 \mathrm{~m} / \mathrm{s}$.

Based on these observations and the research of Lee et al. (2016a, b) and de Goede et al. (2019), we propose a parametrisation of the spreading factor in terms of the contact angle and the Weber number. This is shown in Fig. 10 where we show that $d_{m}$ is a function of the Weber number and the dynamic contact angle through the scaling:

$d_{m}\left(W e, \theta_{D A}\right)= \begin{cases}W e^{1 / 4}\left[1-\cos ^{2}\left(\theta_{D A}\right)\right], & \text { if } \theta_{D A}>90^{\circ} \\ W e^{1 / 4}\left[1+\cos ^{2}\left(\theta_{D A}\right)\right], & \text { if } \theta_{D A}<90^{\circ}\end{cases}$

where $\theta_{D A}$ is the average asymptotic contact angle in the range $0.5<u_{c l}<3.0 \mathrm{~m} / \mathrm{s}$. As seen in Fig. 10, this scaling is also in agreement with the results by Vadillo et al. (2009). Regarding the influence of the droplet size $D_{0}$ on the spreading factor $d_{m}$, we see this effect in Experiments 2 and 3, and 7 and 8, where the droplet size does not to affect $d_{m}$. We clarify that our results are limited to droplet sizes in the millimetre size range, so our observation is in agreement with Visser et al. (2015).

\section{Conclusions}

This paper presents a discussion of various experimental aspects influencing the measurement of the contact angle. We found that the length domain and the order of the polynomial fittings are key parameters on the reliability of the 
measurements of the dynamic contact angle. We found that second-order polynomial fittings produce robust results as they show the lowest standard deviation within other polynomial fittings. Additionally, we found that the optimum domain range for the fittings lies within droplet profile lengths equivalent to $4 \%$ to $10 \%$ of that of the original droplet diameter. In fact, with these conditions the errors in the static contact angle and the dynamic contact angle are 0.6 and $3^{\circ}-5^{\circ}$, respectively. We also show that the correct detection of the contact line is critical to the correct measurement of the contact angle. We argue that our study is important to provide consistency in the fitting method, especially when contrasting experiments and models.

Under our optimised protocols, we conducted several experiments to assess the relevance of the Weber and Reynolds numbers on the contact angle and the maximum spreading diameter. We conclude that, within the impact velocities explored here, the Reynolds number affects both the contact line dynamics and the maximum spreading diameter and can be parametrised by $\theta_{D A}$. Finally, we propose a parametrisation including the We number, the contact angle and the spreading factor that is in agreement with our data and that from Vadillo et al. (2009).

Acknowledgements M. A. Q.-S. acknowledges the funding from the Mexican Energy Ministry (SENER) and the National Council for Science and Technology (CONACyT). AACP is funded by the Royal Society through a University Research Fellowship URF/R/180016 and an Enhancement Grant No. RGF/EA/181002 and via the EPSRC (UK) - CBET (USA) Grant Number EP/S029966/1.

\section{Compliance with ethical standards}

Conflict of interest There are no conflicts to declare.

Open Access This article is licensed under a Creative Commons Attribution 4.0 International License, which permits use, sharing, adaptation, distribution and reproduction in any medium or format, as long as you give appropriate credit to the original author(s) and the source, provide a link to the Creative Commons licence, and indicate if changes were made. The images or other third party material in this article are included in the article's Creative Commons licence, unless indicated otherwise in a credit line to the material. If material is not included in the article's Creative Commons licence and your intended use is not permitted by statutory regulation or exceeds the permitted use, you will need to obtain permission directly from the copyright holder. To view a copy of this licence, visit http://creativecommons.org/licenses/by/4.0/.

\section{References}

Andersen NK, Taboryski R (2017) Drop shape analysis for determination of dynamic contact angles by double sided elliptical fitting method. Meas Sci Technol 28(4):047003

Atefi E, Mann JA Jr, Tavana H (2013) A robust polynomial fitting approach for contact angle measurements. Langmuir 29(19):5677-5688
Bateni A, Susnar S, Amirfazli A, Neumann A (2003) A high-accuracy polynomial fitting approach to determine contact angles. Colloids Surf A Physicochem Eng Asp 219(1-3):215-231

Bayer IS, Megaridis CM (2006) Contact angle dynamics in droplets impacting on flat surfaces with different wetting characteristics. J Fluid Mech 558:415-449

Bergeron V, Bonn D, Martin JY, Vovelle L (2000) Controlling droplet deposition with polymer additives. Nature 405(6788):772

Biolè D, Bertola V (2015) A goniometric mask to measure contact angles from digital images of liquid drops. Colloids Surf A Physicochem Eng Asp 467:149-156

Bonn D, Eggers J, Indekeu J, Meunier J, Rolley E (2009) Wetting and spreading. Rev Mod Phys 81(2):739

Castrejón-Pita J, Martin G, Hoath S, Hutchings I (2008) A simple large-scale droplet generator for studies of inkjet printing. Rev Sci Instrum 79(7):075108

Chen L (2013) Dynamic wetting by viscous liquids: effects of softness, wettability and curvature of the substrate and influence of external electric fields. Ph.D. thesis, University of Darmstadt

Chen H, Muros-Cobos JL, Amirfazli A (2018) Contact angle measurement with a smartphone. Rev Sci Instrum 89(3):035117

Chini SF, Amirfazli A (2011) A method for measuring contact angle of asymmetric and symmetric drops. Colloids Surf A Physicochem Eng Asp 388(1-3):29-37

de Goede TC, de Bruin KG, Shahidzadeh N, Bonn D (2019) Predicting the maximum spreading of a liquid drop impacting on a solid surface: effect of surface tension and entrapped air layer. Phys Rev Fluids 4(5):053602

Del Rio O, Neumann A (1997) Axisymmetric drop shape analysis: computational methods for the measurement of interfacial properties from the shape and dimensions of pendant and sessile drops. J Colloid Interface Sci 196(2):136-147

Derby B (2010) Inkjet printing of functional and structural materials: fluid property requirements, feature stability, and resolution. Annu Rev Mater Res 40:395-414

Eggers J, Fontelos MA, Josserand C, Zaleski S (2010) Drop dynamics after impact on a solid wall: theory and simulations. Phys Fluids 22(6):062101

Eral H, Oh J et al (2013) Contact angle hysteresis: a review of fundamentals and applications. Colloid Polym Sci 291(2):247-260

Fogliati M, Fontana D, Garbero M, Vanni M, Baldi G, Donde R (2006) CFD simulation of paint deposition in an air spray process. JCT Res 3(2):117-125

Good RJ (1992) Contact angle, wetting, and adhesion: a critical review. J Adhesion Sci Technol 6(12):1269-1302

Huhtamäki T, Tian X, Korhonen JT, Ras RH (2018) Surface-wetting characterization using contact-angle measurements. Nat Protocols 13(7):1521-1538

Joanny J, De Gennes P-G (1984) A model for contact angle hysteresis. J Chem Phys 81(1):552-562

Josserand C, Thoroddsen ST (2016) Drop impact on a solid surface. Annu Rev Fluid Mech 48:365-391

Laan N, de Bruin KG, Bartolo D, Josserand C, Bonn D (2014) Maximum diameter of impacting liquid droplets. Phys Rev Appl 2(4):044018

Lamour G, Hamraoui A, Buvailo A, Xing Y, Keuleyan S, Prakash V, Eftekhari-Bafrooei A, Borguet E (2010) Contact angle measurements using a simplified experimental setup. J Chem Educ 87(12):1403-1407

Lee J, Laan N, de Bruin K, Skantzaris G, Shahidzadeh N, Derome D, Carmeliet J, Bonn D (2016a) Universal rescaling of drop impact on smooth and rough surfaces. J Fluid Mech 786:R4

Lee JB, Derome D, Guyer R, Carmeliet J (2016b) Modeling the maximum spreading of liquid droplets impacting wetting and nonwetting surfaces. Langmuir 32(5):1299-1308 
Liu J, Zhu C, Liu K, Jiang Y, Song Y, Francisco JS, Zeng XC, Wang J (2017) Distinct ice patterns on solid surfaces with various wettabilities. Proc Natl Acad Sci 114(43):11285-11290

Lunkad SF, Buwa VV, Nigam K (2007) Numerical simulations of drop impact and spreading on horizontal and inclined surfaces. Chem Eng Sci 62(24):7214-7224

Marmur A (1994) Thermodynamic aspects of contact angle hysteresis. Adv Colloid Interface Sci 50:121-141

Otsu N (1979) A threshold selection method from gray-level histograms. IEEE Trans Syst Man Cybernet 9(1):62-66

Pasandideh-Fard M, Chandra S, Mostaghimi J (2002) A three-dimensional model of droplet impact and solidification. Int J Heat Mass Transf 45(11):2229-2242

Quetzeri-Santiago MA, Castrejón-Pita AA, Castrejón-Pita JR (2019a) The effect of surface roughness on the contact line and splashing dynamics of impacting droplets. Sci Rep 9(1):1-10

Quetzeri-Santiago MA, Yokoi K, Castrejón-Pita AA, Castrejón-Pita JR (2019b) Role of the dynamic contact angle on splashing. Phys Rev Lett 122(22):228001

Rioboo R, Tropea C, Marengo M (2001) Outcomes from a drop impact on solid surfaces. Atomization Sprays 11(2):155-165

Rioboo R, Marengo M, Tropea C (2002) Time evolution of liquid drop impact onto solid, dry surfaces. Exp Fluids 33(1):112-124

Rotenberg Y, Boruvka L, Neumann A (1983) Determination of surface tension and contact angle from the shapes of axisymmetric fluid interfaces. J Colloid Interface Sci 93(1):169-183

Šikalo Š, Marengo M, Tropea C, Ganić E (2002) Analysis of impact of droplets on horizontal surfaces. Exp Therm Fluid Sci 25(7):503-510

Šikalo Š, Wilhelm H-D, Roisman I, Jakirlić S, Tropea C (2005) Dynamic contact angle of spreading droplets: experiments and simulations. Phys Fluids 17(6):062103
Snoeijer JH, Andreotti B (2013) Moving contact lines: scales, regimes, and dynamical transitions. Annu Rev Fluid Mech 45:269-292

Thoraval M-J, Takehara K, Etoh T, Thoroddsen ST (2013) Drop impact entrapment of bubble rings. J Fluid Mech 724:234-258

Vadillo D, Soucemarianadin A, Delattre C, Roux D (2009) Dynamic contact angle effects onto the maximum drop impact spreading on solid surfaces. Phys Fluids 21(12):122002

Visser CW, Frommhold PE, Wildeman S, Mettin R, Lohse D, Sun C (2015) Dynamics of high-speed micro-drop impact: numerical simulations and experiments at frame-to-frame times below 100 ns. Soft Matter 11(9):1708-1722

Yarin A (2006) Drop impact dynamics: splashing, spreading, receding, bouncing. Annu Rev Fluid Mech 38:159-192

Yokoi K, Vadillo D, Hinch J, Hutchings I (2009) Numerical studies of the influence of the dynamic contact angle on a droplet impacting on a dry surface. Phys Fluids 21(7):072102

Yuan Y, Lee TR (2013) Contact angle and wetting properties. In: Bracco G, Holst B (eds) Surface science techniques. Springer series in surface sciences, vol 51. Springer, Berlin, Heidelberg, pp 3-34. https://doi.org/10.1007/978-3-642-34243-1

Zhang G, Quetzeri-Santiago MA, Stone CA, Botto L, Castrejón-Pita JR (2018) Droplet impact dynamics on textiles. Soft Matter 14(40):8182-8190

Publisher's Note Springer Nature remains neutral with regard to jurisdictional claims in published maps and institutional affiliations. 